\title{
BATSE OBSERVATIONS OF NOVA MUSCAE 1991
}

\author{
W. S. Paciesas, M. S. Briggs, G. N. Pendleton \\ University of Alabama in Huntsville
B.A. Harmon, C.A. Wilson, S.N. Zhang, ${ }^{1}$ G. J. Fishman
NASA/Marshall Space Flight Center

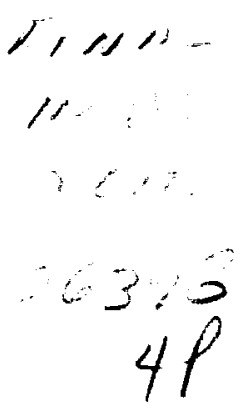

The Compton Burst and Transient Source Experiment (BATSE) detected hard x-ray flux from Nova Muscae 1991 (GS/GRS 1124-68) during an interval of $\sim 100$ days beginning $\sim 130$ days after the January 1991 main outburst. The light curve during this secondary outburst is roughly symmetric, reaching a maximum around mid-July 1991 at an intensity of $\sim 15 \%$ of the peak intensity during the main outburst. The hard $x$-ray spectrum displays a soft-to-hard evolution during the rise to maximum; the post-maximum spectral evolution is less well determined. We compare our observations with those of the GRANAT/SIGMA experiment, which covered the initial outburst well but missed most of the secondary outburst.

\section{INTRODUCTION}

Nova Muscae 1991 was discovered independently as an $\mathrm{x}$-ray transient (GS/GRS 1124-68) in January 1991 by monitors on Ginga (Makino \& the Ginga team 1991) and GRANAT (Lund \& Brandt 1991). In low-energy x-rays $(E<20$ $\mathrm{keV}$ ), the source reached a maximum intensity of $\sim 8 \mathrm{Crab}$ on 15 January (Kitamoto et al. 1992). Subsequently, it decayed almost exponentially with a time constant of $\sim 31$ days for $\sim 75$ days, at which time a secondary outburst occurred, followed by a further exponential decay with a slightly longer time constant $(\sim 37$ days). The power density spectrum showed flat low-frequency noise (below $\sim 2$ $\mathrm{Hz})$ and variable-frequency quasi-periodic oscillations at higher frequencies $(\sim 3-$ $10 \mathrm{~Hz}$ ) (Tanaka, Makino \& Dotani 1991; Grebenev et al. 1992). The spectrum was "ultrasoft" at the lowest energies with an apparently power-law tail extending to several hundred $\mathrm{keV}$ (Gil'fanov et al. 1991). A narrow emission line at $481 \pm 22 \mathrm{keV}$ was detected during a 13 hour SIGMA observation on 20-21 January; this was interpreted by Gil'fanov et al. as a red-shifted positron annihilation line. The power-law tail diminished in intensity faster than the ultrasoft component during the first few months. Nova Muscae was not detected in highenergy x-rays in April, but was present during SIGMA observations in May and August at $\sim 10 \%$ of the January maximum.

An optical nova was detected within the x-ray error circle (Della Valle, Jarvis \& West 1991a, 1991b). Subsequent monitoring showed it to decay at a rate similar to the low-energy x-rays. A binary orbital period of 10.42 hours was found, from which a mass function of $3.1 \pm 0.4 \mathrm{M}_{\odot}$ was determined (McClintock, Bailyn \& Remillard 1992), making the source a leading black hole candidate.

The BATSE instrument on the Compton Gamma Ray Observatory

\footnotetext{
1 Also Universities Space Research Association
} 
(CGRO) functions as an all-sky monitor by detecting Earth occultations of low-energy gamma-ray sources (Harmon et al. 1992). CGRO was launched in April 1991, too late to observe the main outburst of Nova Muscae. Nevertheless, we used the occultation method to search for subsequent emission and found that Nova Muscae was detectable by BATSE during an interval of $~ 100$ days, beginning just before the May 1991 detection by SIGMA.

\section{OBSERVATIONS}

The BATSE Earth occultation methodology has been described by Harmon et al. (1992). We obtained spectra in 16 energy channels spanning the range $20-2000 \mathrm{keV}$ by measuring differences in detector counting rates as Nova Muscae rose above or set below the horizon. We fit the data for each occultation in each energy channel with a model consisting of a source term superimposed on a time-varying background. Potential interference from other sources was handled as follows: A catalog of known bright or potentially bright sources is maintained as part of BATSE mission operations. All such sources whose location was within $60^{\circ}$ of the axis of any detector which also saw Nova Muscae were treated as possible interfering sources. If the occultation time of any of the possible interfering sources fell within $10 \mathrm{~s}$ of a Nova Muscae occultation step, that step was eliminated from further analysis. Otherwise, terms were included in the fit for all possible interfering sources with an occultation step within the fit region ( $\pm 110 \mathrm{~s}$ around each Nova Muscae occultation step).

We searched for emission from Nova Muscae during the interval 28 April31 October 1991 (TJD 8374-8560). However, during portions of this time, including the interval prior to $5 \mathrm{May}$, the source was too far out of the CGRO orbit plane to produce usable occultations. Nevertheless, it was clear from inspection of the initial results that a significant signal was detected from Nova Muscae during much of the time when usable occultations were available. In order to produce a light curve, we summed the individual occultation count rates over 3 day intervals and deconvolved the spectra using conventional forward-folding. For this purpose we assumed a power-law input spectrum with fixed number index $\alpha=-2$.

The upper panel of Figure 1 shows the light curve derived from the BATSE data. The temporal evolution is characterized by a slow, approximately linear rise beginning around 15 May, leading to a single broad, symmetric maximum around $13 \mathrm{July}$, and a decay below our detection limit around 1 September. For comparison, we show in the same figure the SIGMA observations (Gil'fanov et al. 1991). It can be seen from the figure that the maximum flux during our observations was $\sim 15 \%$ of the primary maximum. We note that the secondary maximum fell approximately midway between the last two SIGMA observations.

We investigated the evolution of the spectrum during the secondary outburst by summing the data over longer intervals (but never longer than one CGRO viewing period, usually two weeks), and then fitting various models to the resulting count spectra. A single power-law model was found to be an adequate fit in all intervals, although alternative models such as optically thin thermal bremsstrahlung and Sunyaev-Titarchuk Comptonization could not in general be ruled out. In the lower panel of Figure 1 we show the evolution of the power-law index during our observations compared with the SIGMA results. During the secondary outburst, the hardness increases as the intensity rises to a maximum; the trend after the maximum is unclear because of limited statistics. Gil'fanov et al. (1991) noted that their data were consistent with a clustering 


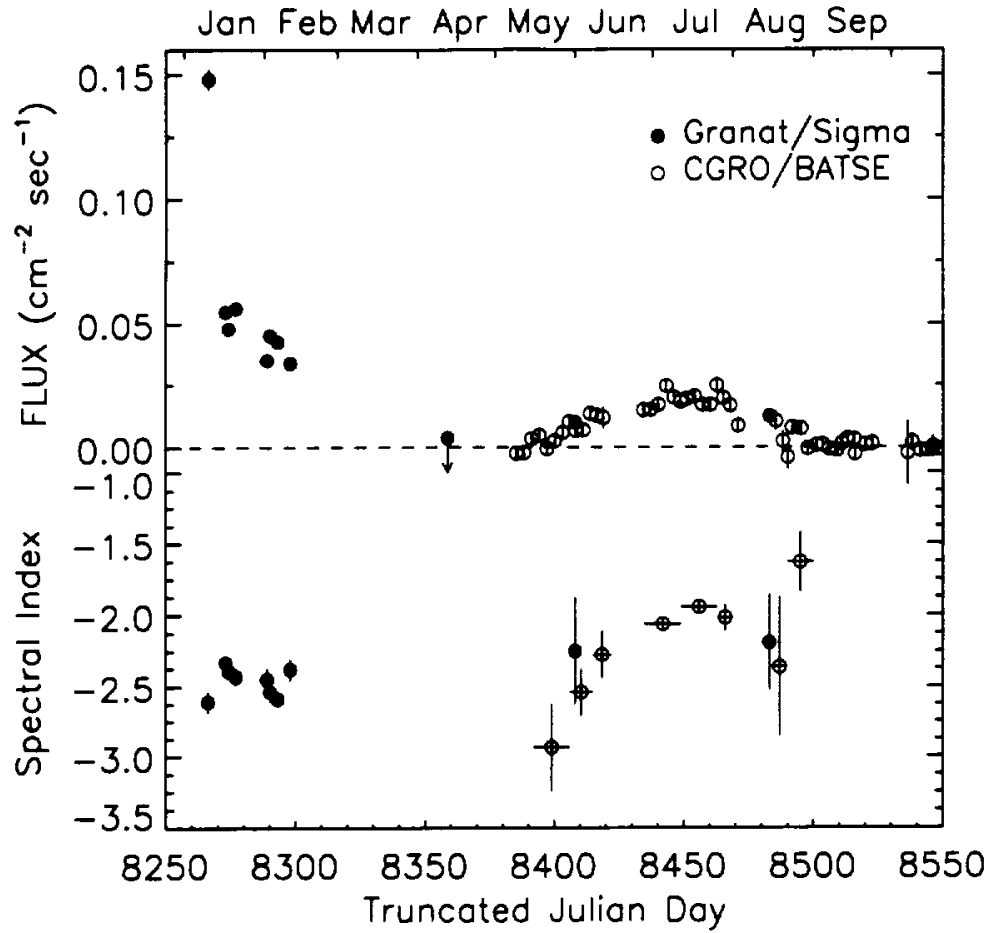

Fig. 1. Intensity and spectral evolution of the entire Nova Muscae outburst, combining both BATSE and SIGMA data, the latter from Gil'fanov et al. 1991. The flux is integrated over $35-100 \mathrm{keV}$.

around an average index $\alpha \approx-2.5$ with no trend throughout the outburst. The BATSE data, however, show a significantly harder spectrum $(\alpha \approx-2)$ around the secondary maximum and possibly an even harder spectrum just before the source disappears (although the latter may be subject to systematic errors).

Recently, a technique for producing images using the BATSE occultation data has been developed (Zhang et al. 1993). We generated a number of images of the region around Nova Muscae to verify that the latter was in fact the source of any emission detected in our occultation fitting, and that all significant nearby sources had been accounted for. Samples of these are shown in Figure 2, from which one can see that no other source besides Nova Muscae is visible above $55 \mathrm{keV}$. Below that energy the only other significant source is the recurrent transient pulsar GX 301-2, which was included in our analysis.

\section{DISCUSSION}

Two main theoretical mechanisms have been proposed to explain the outbursts of black-hole X-ray novae: the disk thermal instability model and the mass transfer instability model (Hameury et al. 1990 \& refs. therein). Chen, Livio \& Gehrels (1993) attempted to explain the entire light curve by a combination of these ideas: a main disk instability outburst is followed by a second outburst (in soft $x$-rays) due to mass transfer by evaporation of the outer layers of the secondary by $x$-ray heating. A third outburst also results from mass transfer, this time due to more prolonged heating and expansion of the secondary's convective layer. The second outburst in Nova Muscae was clearly seen in late March in soft x-rays but not in hard x-rays (Kitamoto et al. 1992). The hard $x$-ray secondary maximum which we observed would then correspond in the above scenario to the third outburst. However, if both of the secondary 

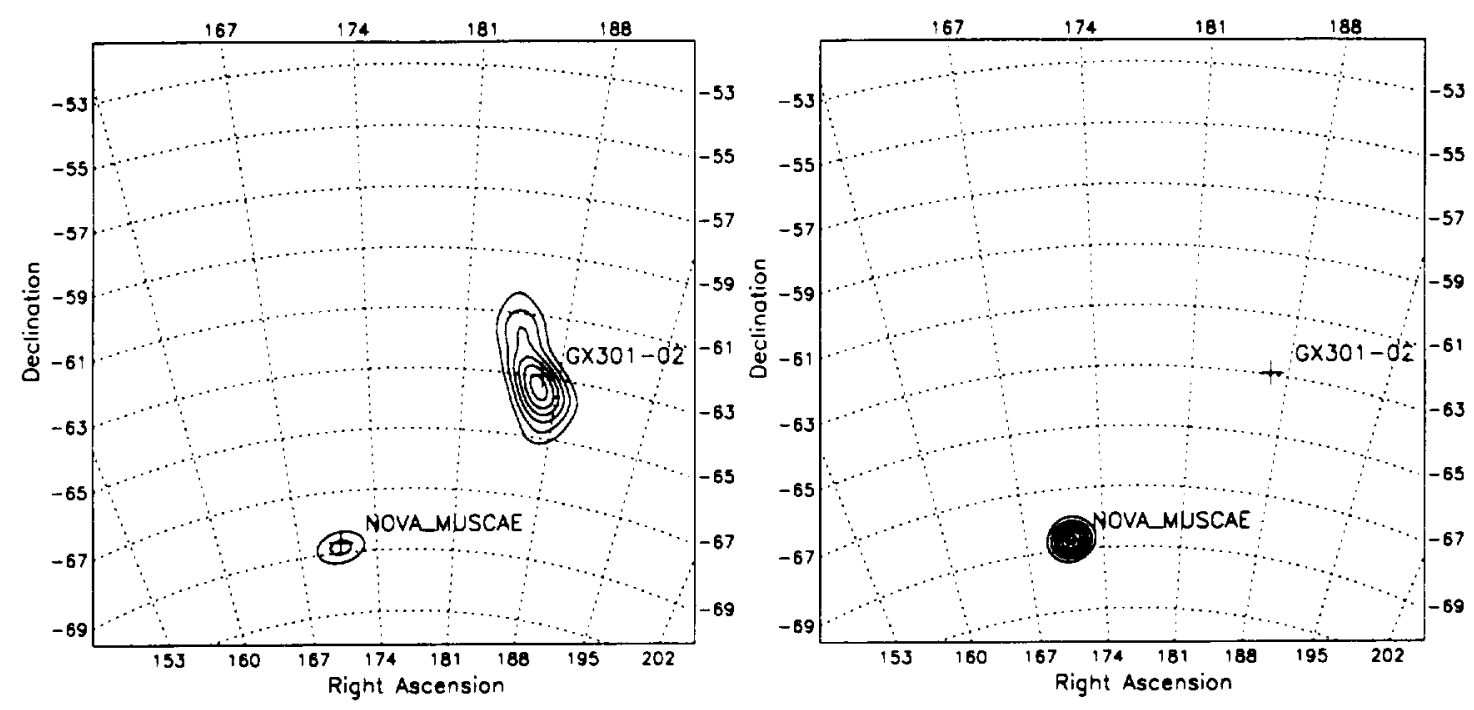

Fig. 2. Images of a region of the sky near Nova Muscae around the time of the secondary maximum (27 June- 7 August). The images were produced using the radon transform and maximum-entropy inversion (Zhang et al. 1993). The energy intervals are $25-55 \mathrm{keV}$ and $55-110 \mathrm{keV}$ for the left and right panels, respectively.

maxima simply represent episodes of increased mass accretion through the disk, then it is necessary to explain their completely different spectral characteristics. Future models must consider the distinctly different temporal behaviors of the hard power-law and ultrasoft spectral components in black-hole $\mathrm{x}$-ray novae.

\section{REFERENCES}

Chen, W., Livio, M., \& Gehrels, N., 1993, ApJ, 408, L5

Della Valle, M., Jarvis, B. J., \& West, R. 1991a, IAU Circ. No. 5165

Della Valle, M., Jarvis, B. J., \& West, R. M. 1991b, Nature, 353, 50

Gil'fanov, M., et al. 1991, Sov. Astron. Lett., 17, 437

Grebenev, S., et al. 1992, Sov. Astron. Lett., 18, 11

Hameury, J.-M., et al. 1990, ApJ, 353, 585

Harmon, B.A., et al. 1992, in The Compton Observatory Science Workshop, ed.

C. R. Shrader, N. Gehrels \& B. Dennis (NASA CP-3137), 69

Kitamoto, S., et al. 1992, ApJ, 394, 609

Lund, S., \& Brandt, S. 1991, IAU Circ. No. 5161

Makino, F., \& the Ginga team 1991, IAU Circ. No. 5161

McClintock, J., Bailyn, C., \& Remillard, R. 1992, IAU Circ. No. 5499

Tanaka, Y., Makino, F., \& Dotani, T. 1991, in Proc. Workshop on Nova Muscae 1991, ed. S. Brandt (Lyngby: Danish Space Research Inst.), 125

Zhang, S. N., Fishman, G. J., Harmon, B. A., \& Paciesas, W. S. 1993, Nature, 366,245 